\title{
Metallo-celluloses, 1 Synthesis and solution properties of 4-ferrocenylbutyl- substituted cellulose derivatives
}

\author{
Bettina Laube, Wolfgang Radke, Matthias Rehahn *, Winfried Wunderlich
}

Ernst-Berl-Institute for Chemical Engineering and Macromolecular Science, Darmstadt University of Technology, Petersenstr. 22, 64287 Darmstadt, Germany; Fax +49 (0)6151/ 16 4670; mrehahn@dki.tu-darmstadt.de

(Received: September 19, 2003; published: December 6, 2003)

\begin{abstract}
An efficient method is described for the simultaneous introduction of 4-ferrocenylbutyl and n-hexyl side groups into cellulose. For this purpose, cellulose triacetate is treated in dimethyl sulfoxide/ $\mathrm{NaOH}$ solution with mixtures of 4-bromobutylferrocene and $n$-hexyl bromide. An almost quantitative conversion of the cellulose hydroxyl groups was reached when less than one ferrocene substituent was attached to the cellulose repeating units on average. At higher degrees of ferrocene substitution, some of the cellulose hydroxyl groups seem to remain unsubstituted. This conclusion has been drawn on the basis of size exclusion chromatography (SEC) with online light-scattering detector where coil contraction was observed with increasing ferrocene content in the cellulose sample. This finding was further supported by IR spectroscopy, viscometry, and conventional SEC investigations. We assume that steric hindrance of the bulky side groups is responsible for incomplete conversion.
\end{abstract}

\section{Introduction}

Cellulose is one of the most abundant renewable carbon sources worldwide, but only a few percent of the material formed by plants every year are used by men [1-3]. In our endeavour to safe fossil resources, much effort has been spent to set the stage for a more extensive use of natural polymers in technique, and to create new products able to compete with classic materials. Here, it is an interesting challenge not only to broaden the scope of well-established large scale applications but to use natural polymers like cellulose as parent compounds for smart materials [1-14]. And indeed, there exist some examples of promising cellulose-based functional polymers in the literature $[1,2]$. Most of them have a very regular substitution pattern and are accessible via rather sophisticated syntheses. Therefore, we assume this first generation of functional cellulose derivatives will succeed on the market only in a few specific cases. For most practically relevant materials, much simpler derivatization processes are mandatory for cost and safety reasons. But such rather straightforward syntheses usually result in substitution patterns of lower regularity. Thus, one has to find strategies leading to materials of lower regularity but nevertheless showing the desired properties. And prior to any broader application of functional cellulose derivatives available via convenient and cheap processes it is necessary to learn more about the specific structure-property relationships of the thus prepared 
materials. Another important consequence of a less regular substitution pattern is that one has to ensure that the properties of the cellulose derivatives do not depend too much on the respective starting material or on the synthetic conditions. To conclude, more extensive use of the still nearly unexploited potential of cellulose as basic material for technically relevant functional polymers requires more efficient macromolecular substitution routes, and asks for a more profound knowledge on the structure-property relationships. Both demands are not easy to achieve because cellulose is a chemically rather unstable material with complex solution behaviour. This makes chemical conversions very sensitive towards even minor changes in the reaction conditions or in the starting material, and minimal alterations often lead to products with clearly different properties. Hence, reliable comparison of experimental results obtained from seemingly identical polymers is often prevented.

Nevertheless, the attractiveness of easily available functional cellulose derivatives is a strong motivation to validate synthetic methods with respect to their potential for the preparation of new polymers based on renewable raw materials. In particular, cellulose derivatives bearing ionic, electro- or photochromic, redox- or catalytically active side groups are of interest. Therefore, we decided to test synthetic procedures with regard to their potential for introducing transition-metal complexes into cellulose. The desired cellulose derivatives should contain freely eligible amounts of these functional side groups, statistically distributed along and among the individual cellulose chains. On the other hand, per-substitution of the cellulose derivatives is essential in order to avoid problems due to hydrogen bonds and to render the materials soluble and processable. Hence, the methods to be selected have to guarantee quantitative conversion of all hydroxyl groups present in cellulose with either the functional metal complex or a second type of substituent $R$ attached to cap the remaining hydroxyl groups ('auxiliary substituents'). After having prepared these polymers in a reproducible way, their properties have to be analyzed in dilute solution as a function of the density of the attached functional side groups.
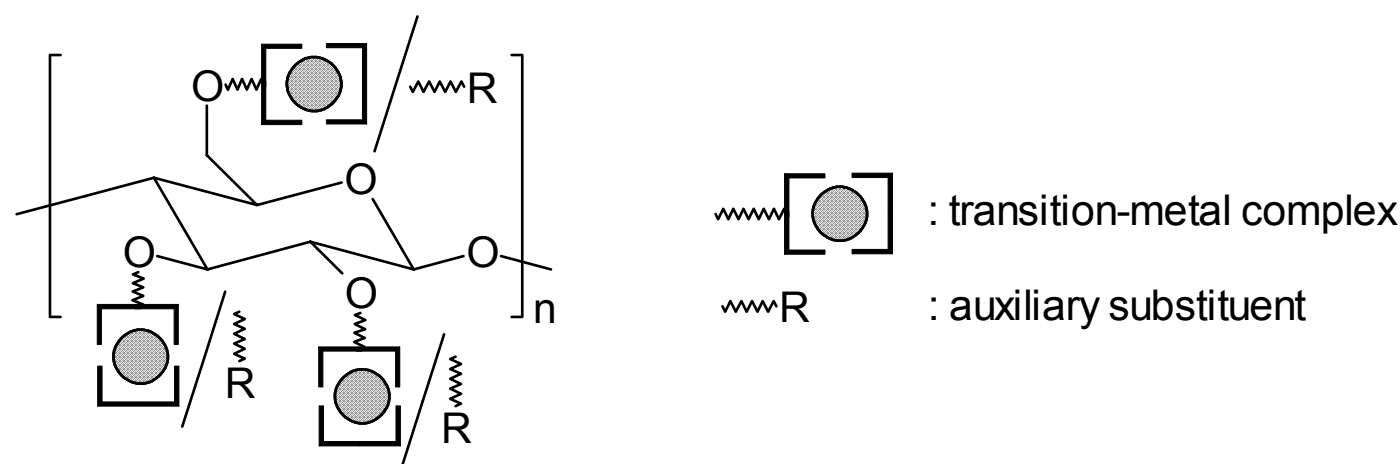

Fig. 1. General formula of the target compounds aimed at in the present work

\section{Strategy}

When a homogeneous distribution of two or more different substituents along and among cellulose chains is aimed at, production of these materials in homogeneous solution is imperative. But homogeneously dissolved cellulose is only available in a few solvents, which are able to break hydrogen bonds very efficiently. However, such solvent systems - concentrated acids [15], strong bases [16-18], complexing [15,1823] or derivatizing media $[15,18,24]$ - are expensive and problematic and thus useful only for specific conversions but not as standard reaction media. An elegant alter- 
native is application of readily soluble per-substituted cellulose derivatives bearing substituents that can be replaced easily and completely by other functional groups [25-30]. It is known, for example, that cellulose triacetate (1) can be transferred into cellulose ethers when treated with $\mathrm{NaOH}$ and organic halides in homogeneous dimethyl sulfoxide (DMSO) solution [25,30]. Hence, it was this procedure that we selected for our project. For the introduction of transition-metal complexes into cellulose via the above way, there are two different possible strategies (Scheme 1).
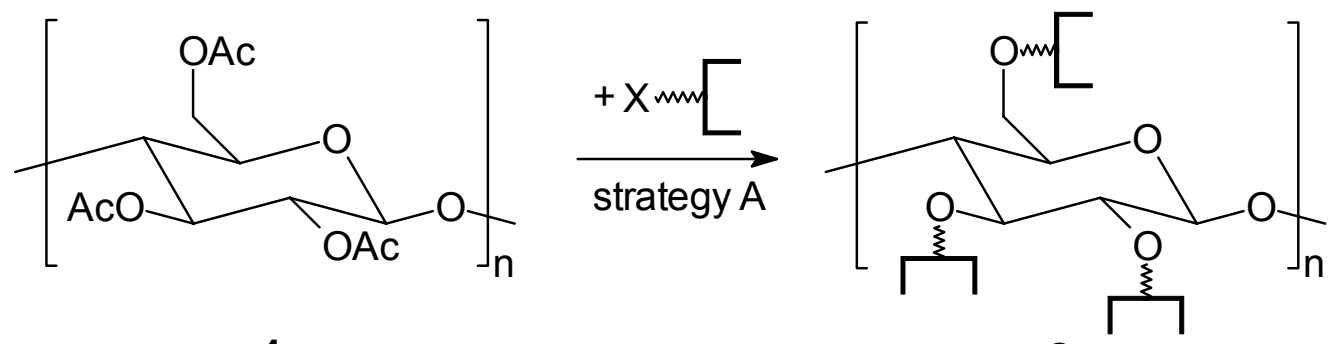

1

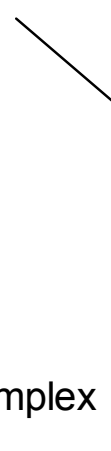

2

Ac: $\mathrm{CH}_{3}-\mathrm{CO}-$

$\mathrm{X}$ : reactive functionality

$[$ : chelating ligand

[O] : transition-metal complex
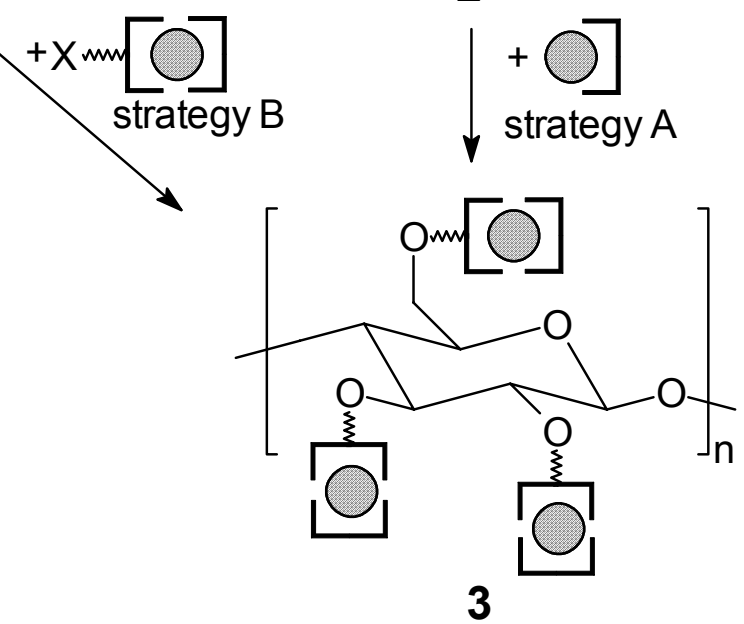

Scheme 1. Schematic representation of strategies A and B leading to transition-metal complex substituted cellulose derivatives

In strategy $\mathrm{B}$, preformed transition-metal complexes are attached to the cellulose backbone, while in strategy A, ligand moieties are introduced first, which were converted into transition-metal complexes in a second macromolecular substitution step. While strategy B is recommended for uncharged and stable transition-metal complexes - labile and/or charged complexes complicate polymer characterization strategy $A$ is the method of choice for chemically unstable and/or charged complexes. Here, polymer characterization can be done after etherification by means of ligand-intermediate $\mathbf{2}$. For the present work we planned to introduce the uncharged and chemically quite inert transition-metal complex ferrocene. Hence, strategy B was the method of choice.

In order to be as free as possible in the variation of the degrees of ferrocene substitution $\left(\mathrm{DS}_{\mathrm{fc}}\right)$ but to nevertheless ensure per-substitution of the cellulose derivatives as a whole $\left(\mathrm{DS}_{0}=3\right)$ - essential to prevent hydrogen bonds formed by free hydroxyl groups, which complicate interpretation of the observed solution properties - conventional side groups ('auxiliary substituents') $R$ had to be attached to the cellulose in addition to the functional transition-metal complexes. These auxiliary substituents must not affect the introduction of the metal complexes but reliably close all the 
remaining hydroxyl groups. Therefore, the reagents $\mathrm{R}-\mathrm{X}$ should have similar reactivity as the metal-containing reagent, and should be applied simultaneously, i.e., in a mixture with the metal-containing reagent (Scheme 2).

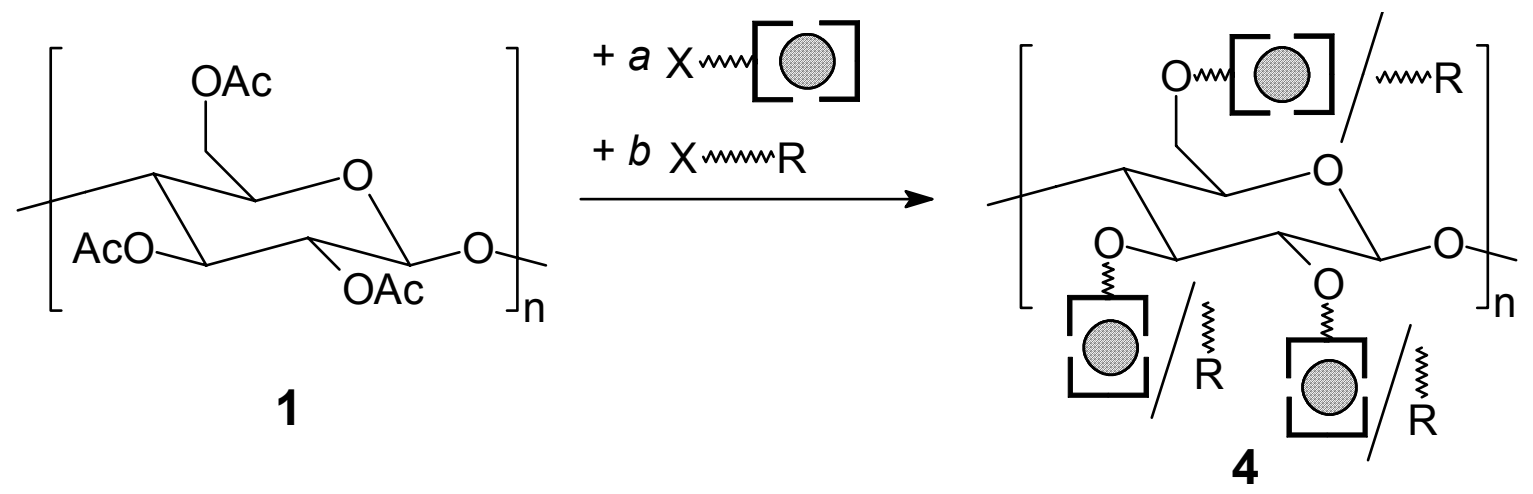

Scheme 2. Introduction of metal complexes and auxiliary substituents $\mathrm{R}$ into cellulose triacetate, 1

According to the literature, alkyl and benzyl halogenides are the most useful reagents for etherification of cellulose triacetate in $\mathrm{DMSO} / \mathrm{NaOH}[25,30]$. In order to prevent adverse effects of the ferrocene moiety on the ether formation, an aliphatic spacer should be placed between the metal complex and the reactive halogen functionality $X$. Hence, 4-ferrocenyl-1-n-butyl bromide $\mathbf{5}$ was selected as the reagent for the introduction of the metal complexes and accordingly, n-hexyl bromide $\mathbf{6}$ was selected as auxiliary reagent since we expected a similar reactivity and a low steric hindrance here. Summing up, the conversion shown in Scheme 3 was the process to be carried out, and $a$ and $b$ should be varied according to (i) $0 \leq a, b \leq 3$ and (ii) $a+b=3$.

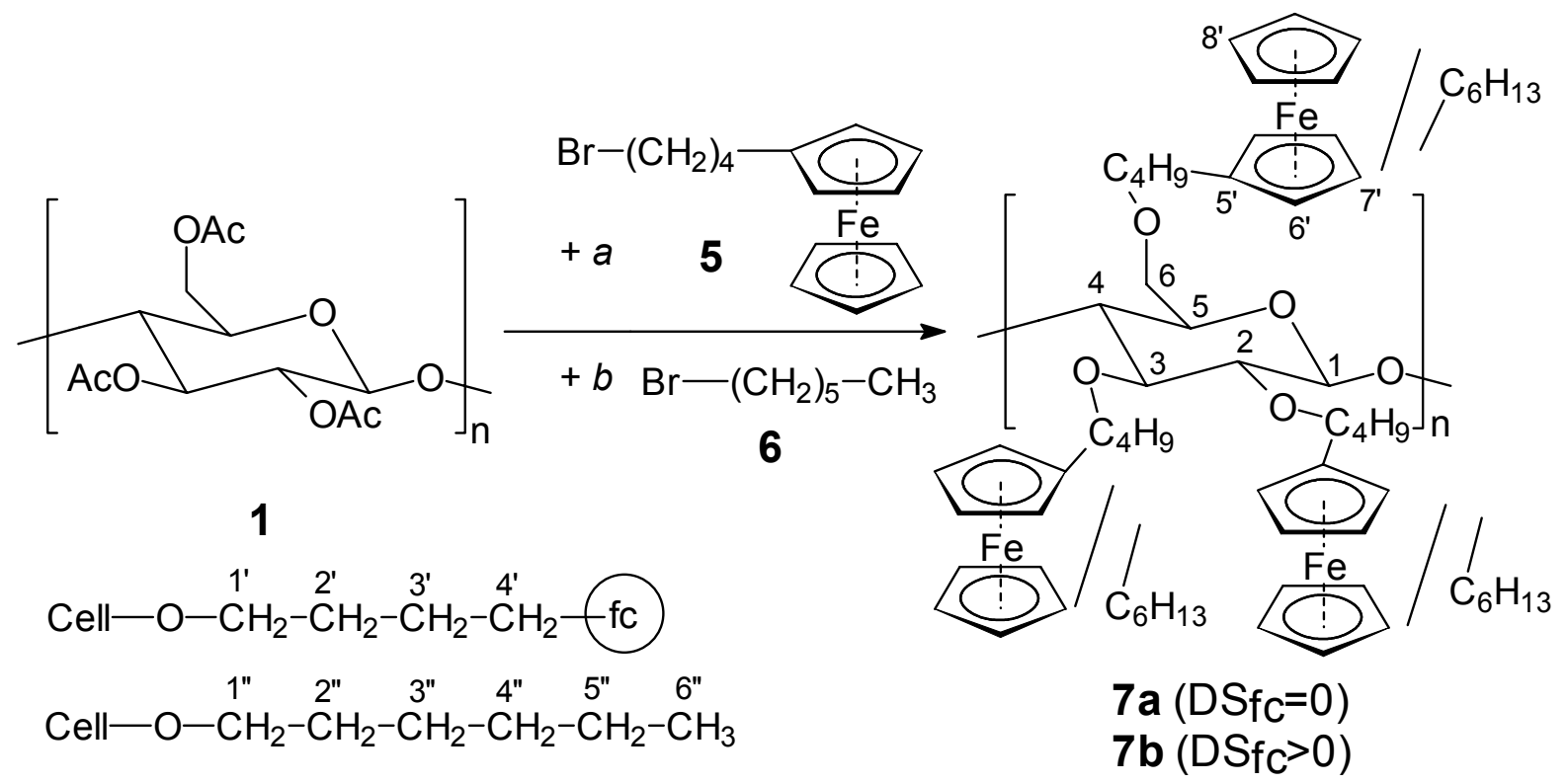

Scheme 3. Derivatization of cellulose triacetate 1 as is studied in this work; the value of $D S_{\text {fc }}$ given in brackets for compounds 7 is the degree of ferrocene substitution; $0 \leq$ $\mathrm{DS}_{\mathrm{fc}} \leq 3$; the given numbering is used for NMR signal assignment 


\section{Results and discussion}

\section{Synthesis of starting materials}

4-Ferrocenyl-1-n-butyl bromide 5 was the required reagent for introducing the ferrocene groups into cellulose triacetate 1 according to strategy $\mathrm{B}$. It was prepared via Friedel-Crafts acylation of ferrocene using 4-bromobutane-1-carboxylic chloride, followed by Clemmensen reduction of the resulting ketone using zinc amalgam [31]. The acylation reaction was possible in $65-70 \%$ yield, the subsequent reduction in yields of $75-80 \%$. Using NMR spectroscopy and mass spectrometry, the constitution and the high purity of the obtained materials was proved.

\section{Model conversions}

Two slightly different procedures are described in the literature for etherification of cellulose triacetate with alkyl bromides. In both cases, cellulose triacetate is first dissolved at $70^{\circ} \mathrm{C}$ for $1 \mathrm{~h}$ in dry DMSO [25,30]. Then, a twelve-fold excess of powdered $\mathrm{NaOH}$ is added, and the solution is stirred for one further hour. Finally, according to Kondo's procedure [25], 12 equiv. of alkyl bromide are added over a period of $5 \mathrm{~h}$ in small portions. Subsequently, the reaction mixture is stirred at $70^{\circ} \mathrm{C}$ for 1 day (method A). According to Wenz et al. [30], on the other hand, 12 equiv. of alkyl bromide are added at once, and the reaction mixture is stirred for 8 days at room temperature (method B).

The aim of the initial model experiments was to find out the optimum reaction conditions for reagents 5 and $\mathbf{6}$ to be used here. Hence, pure n-hexyl bromide $\mathbf{6}$ was applied as reagent first, leading to polymers $7 \mathbf{a}$. It could be shown that both procedures give rubber-like, slightly yellowish and readily soluble cellulose derivatives 7 a in $>80 \%$ after work-up - provided carefully powdered and dry $\mathrm{NaOH}$ is used. If, on the other hand, the conditions were not properly chosen, products insoluble in solvents like tetrahydrofuran (THF) or chloroform were obtained. This insolubility was assumed to be due to incomplete substitution of the hydroxyl groups, leading to the re-formation of some (intra- and intermolecular) hydrogen bonds and thus to crosslinking. Hence, solubility is a good first test for an (almost) complete conversion.

The products obtained under best conditions were characterized subsequently using NMR and IR spectroscopy. IR spectroscopy shows all the expected absorptions for $\mathrm{n}$-hexyl cellulose. Moreover, there was only a weak absorption around $3400 \mathrm{~cm}^{-1}$ in some samples, which might point towards remaining cellulose hydroxyl groups. Alternatively, it could be caused by moisture from the work-up procedure. And indeed, further drying at $40-50^{\circ} \mathrm{C}$ under high vacuum resulted in the complete disappearance of this latter absorption. Hence, it was concluded that the origin of this absorption was some water held back in the sample due to its rubber-like consistence, and both methods seemed to give per-substituted n-hexyl cellulose $7 a$ of equal quality.

In the next step we analyzed whether the given reaction times might be shortened. In particular 8 days seemed to be quite long. To monitor the progress of conversions, representative samples were taken from the reaction mixtures at regular intervals, and the formed cellulose derivatives were studied after isolation using IR spectroscopy. It was found that the reaction times given in the literature are indeed needed to ensure quantitative conversion of all cellulose hydroxyl groups: only after that times no absorptions were observed any more in the $3400 \mathrm{~cm}^{-1}$ region. 
NMR spectroscopy supported the above conclusions: signal patterns were obtained in both cases that were characteristic of constitutionally homogeneous n-hexyl cellulose, and no hints were found in these spectra for free hydroxyl groups or side reactions. However, for detecting chain degradation, neither IR nor NMR spectroscopy are the methods of choice because of their limited sensitivity. Therefore, SEC was applied to compare the products with regard to their chain lengths. Direct comparison of the SEC traces was possible since for all model reactions the same batch of cellulose triacetate 1 was used. Two representative SEC traces of n-hexyl celluloses $7 \mathrm{a}$ are shown in Fig. 2, once prepared according to method $A$, once by method B. A monomodal molar mass distribution is evident in both cases but with clear differences in the molar masses: it was a reproducible result that materials obtained at $70^{\circ} \mathrm{C}$ according to method $A$ had clearly lower molar masses as compared to those prepared at room temperature via method B. Depolymerization of the cellulose backbone is obviously accelerated at elevated temperatures.

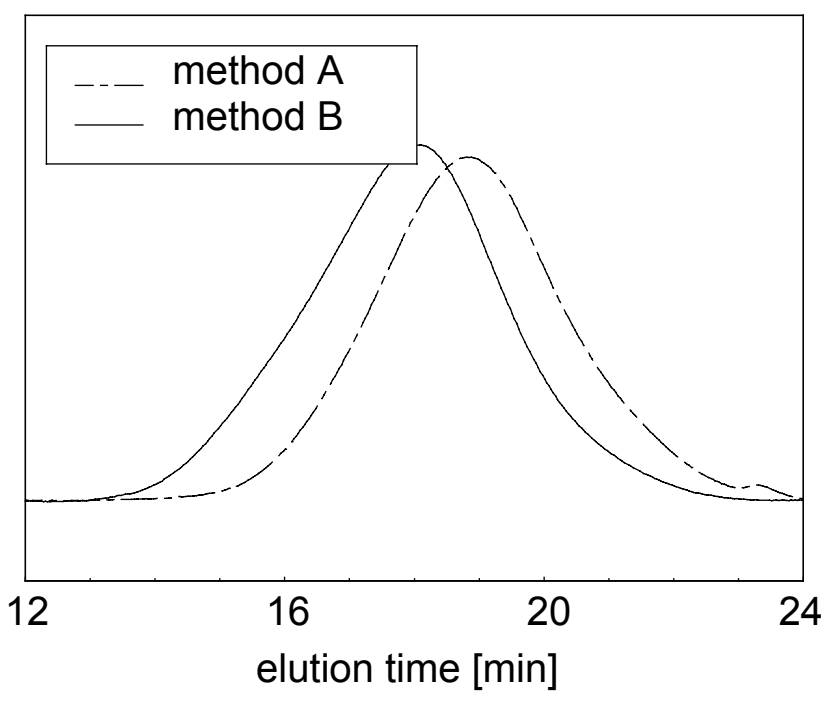

Fig. 2. SEC traces of $n$-hexyl celluloses $7 a$, prepared according to methods $A$ and $B$, respectively. SEC traces were recorded at $30^{\circ} \mathrm{C}$ in THF

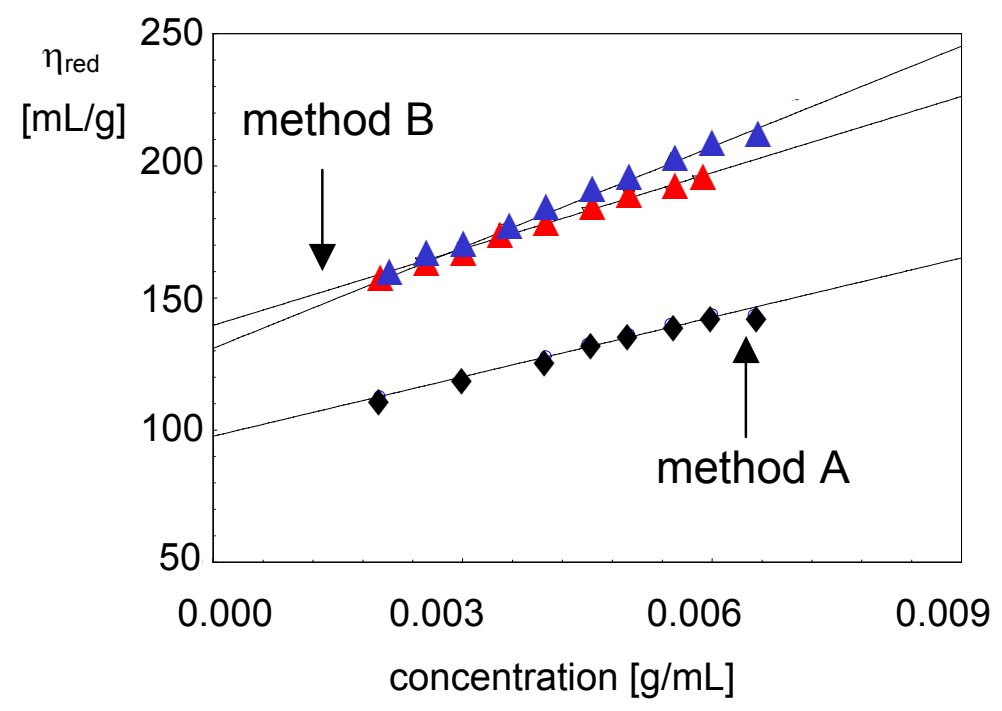

Fig. 3. Huggins plots of three samples of $n$-hexyl cellulose $7 a$, prepared according to methods $\mathrm{A}$ and $\mathrm{B}$, respectively; measured in $\mathrm{CHCl}_{3}$ at $30^{\circ} \mathrm{C}$ 
Viscosity studies as well are useful to compare degrees of polymerization of otherwise identical polymers. Viscometry reconfirmed the results obtained by SEC: $n$-hexyl cellulose samples prepared at $70^{\circ} \mathrm{C}$ showed lower intrinsic viscosities as compared to those obtained at room temperature (Fig. 3). Hence, we decided to use method B for the preparation of ferrocene-containing cellulose derivatives $7 \mathrm{~b}$ because both, SEC and viscometry, showed the samples prepared at room temperature to have clearly higher degrees of polymerization.

\section{Synthesis of the 4-ferrocenyl-n-butyl- and n-hexyl-substituted cellulose ethers}

For the simultaneous introduction of 4-ferrocenyl-n-butyl and n-hexyl side groups into the cellulose backbone, cellulose triacetate 1 was treated according to method $B$ with mixtures of 4-ferrocenyl-n-butylbromide 5 and n-hexyl bromide 6 (see Scheme 4). The composition of the reagent mixture, i.e., $\mathbf{5}: \mathbf{6}$, was varied over a wide range (see below). During the reactions, the originally orange colour of the mixtures became darker but the mixtures remained homogeneous even after full reaction time. Only in those cases where very high amounts of 5 were applied some few gel particles were observed in the reaction vessel. Then, the products were isolated and carefully dried. Orange rubbers (low $\mathrm{DS}_{\mathrm{fc}}$ ) or powders (higher $\mathrm{DS}_{\mathrm{fc}}$ ) were obtained in most cases except those where pure $\mathbf{5}$ was used as the reagent, i.e., which should have degrees of ferrocene substitution of $\mathrm{DS}_{\mathrm{fc}}=3$. These latter materials changed their colour during the work-up from orange-brown into greenish-black and became insoluble in all solvents. Partial insolubility after work-up - but no discolouring - was also observed for products where a value of $\mathrm{DS}_{\mathrm{fc}} \geq 1.5$ was adjusted. Finally, all the materials were analyzed using IR and NMR.

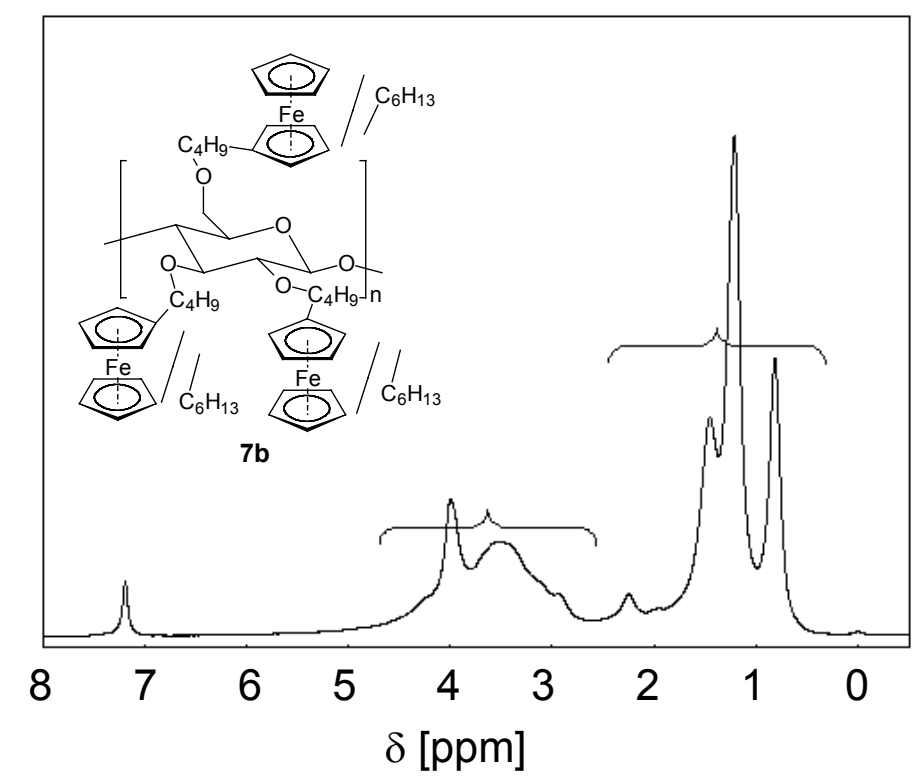

Fig. 4. ${ }^{1} \mathrm{H}$ NMR spectrum of cellulose derivative $7 \mathrm{~b}(1)$; the spectrum was recorded in $\mathrm{CDCl}_{3}$ at room temperature

The proton NMR spectra of polymers $\mathbf{7 b}$ show two characteristic groups of absorptions (Fig. 4): at $\delta \approx 2.6-4.6 \mathrm{ppm}$ there is a very broad and unstructured signal. Here, the protons of the cellulose backbone absorb as well as the $\alpha-\mathrm{CH}_{2}$ protons of the n-butyl spacer and of the n-hexyl side chains. Moreover, there is a 
clearly detectable peak at $\delta \approx 4$ ppm where the ferrocene protons appear. In the second group of absorptions, i.e., in the $\delta \approx 0.8-2.5 \mathrm{ppm}$ region, there are the signals of all remaining protons of the $n$-hexyl side chains and of the n-butyl spacers. The intensity of the two signal groups allows estimation of the ratio of the different substituents present in the product. The determined values are given in Tab. 1 together with the reagent ratio used for the respective entry. Comparison of these data shows that the calculated and the experimentally observed ratios of the substituents agree quite well. This proves a very similar reactivity of the two bromoalkyl reagents 5 and 6 under the applied conditions. In Fig. 5, the results are represented graphically.

Tab. 1. Values of $\mathrm{DS}_{\mathrm{fc}}$ (expected and determined by NMR), refractive index increments (in THF), and average values of $M_{w}$ of cellulose derivatives 7 , determined by SEC using an online light-scattering detector (SEC-MALLS)

\begin{tabular}{ccccc}
\hline Sample & $\mathrm{DS}_{\mathrm{fc}}($ calc. $)$ & $\mathrm{DS}_{\mathrm{fc}}(\mathrm{NMR})$ & $\mathrm{d} n / \mathrm{d} c$ & $M_{\mathrm{w}}[\mathrm{g} / \mathrm{mol}]$ \\
\hline $\mathbf{7 a}$ & 0 & 0 & 0.067 & 200000 \\
$\mathbf{7 b}(1)$ & 0.22 & 0.2 & 0.069 & not determ. \\
$\mathbf{7 b}(2)$ & 0.26 & 0.2 & not determ. & 127000 \\
$\mathbf{7 b}(3)$ & 0.46 & 0.5 & 0.075 & 354000 \\
$\mathbf{7 b}(4)$ & 1.05 & 1.0 & 0.080 & 481000 \\
$\mathbf{7 b}(5)$ & 1.74 & 1.4 & not determ. & 253000 \\
$\mathbf{7 b}(6)$ & 2.45 & 1.8 & not determ. & 105000 \\
$\mathbf{7 b}(7)$ & 2.84 & 2.3 & 0.102 & 226000
\end{tabular}

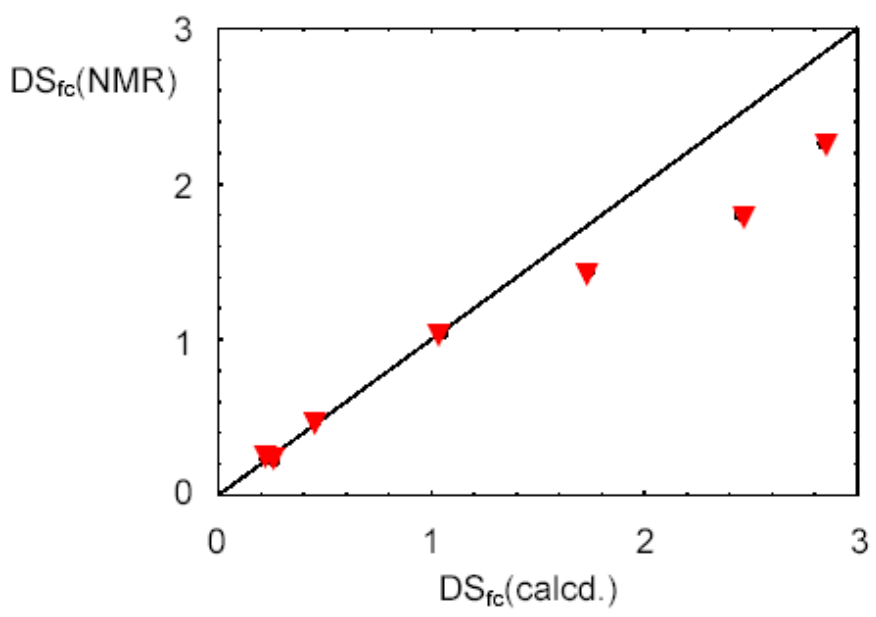

Fig. 5. Experimentally determined $\left({ }^{1} \mathrm{H} N M R\right) \mathrm{DS}_{\mathrm{fc}}$ values of cellulose derivatives $\mathbf{7 b}$, plotted $v s$. the expected values of $\mathrm{DS}_{\mathrm{fc}}$

A deviation from linearity is only remarkable for values of $D S_{f c} \geq 1.5$. Starting with this value of $D S_{f c}$, also some insoluble product fractions were observed. The highest value of $\mathrm{DS}_{\mathrm{fc}}$ achieved under the applied conditions was 2.3. Realization of a $\mathrm{DS}_{\mathrm{fc}}=$ 3 was not possible because only insoluble and discoloured products were obtained. This might be due to the steric conflicts of the ferrocene substituents at the cellulose 
backbone, preventing the formation of a too high density of these substituents along the chains, and may be also prevent complete substitution $\left(\mathrm{DS}_{\mathrm{fc}}<3\right.$ ): insolubility of (parts of) these products points towards remaining hydroxyl groups. Moreover, because cellulose derivatives having a $\mathrm{DS}_{\mathrm{fc}} \approx 3$ discolour during work-up, there might occur some redox processes - caused maybe due to the stabilization of mixed-valent states - which additionally prevent re-dissolution of the isolated materials. However, no spectroscopic evidence was found for redox processes in cellulose derivatives $\mathbf{7 b}$ having values of $\mathrm{DS}_{\mathrm{fc}}$ lower than approx. 2.8. Also, the proton NMR spectra did not show any evidence for free hydroxyl groups but this statement is valid only for the soluble fraction and is moreover rather weak due to the low sensitivity of the NMR method towards such groups: it is reliable to assume that - if there are unsubstituted hydroxyl groups in the product - these moieties are preferentially in the insoluble part. For that part of the product, more detailed information is available using IR spectroscopy (see below).

In ${ }^{13} \mathrm{C}$ NMR spectra as well, all absorptions were found which are expected for the desired products. In addition to resonances of the cellulose backbone and of the alkyl groups, the absorptions of the $\mathrm{C}-\mathrm{H}$ carbons of the ferrocene moieties absorb around $\delta \approx 68 \mathrm{ppm}$ while their quaternary carbon absorbs at $\delta \approx 89.08 \mathrm{ppm}$. For clarity, a DEPT spectrum is shown as an example in Fig. 6.

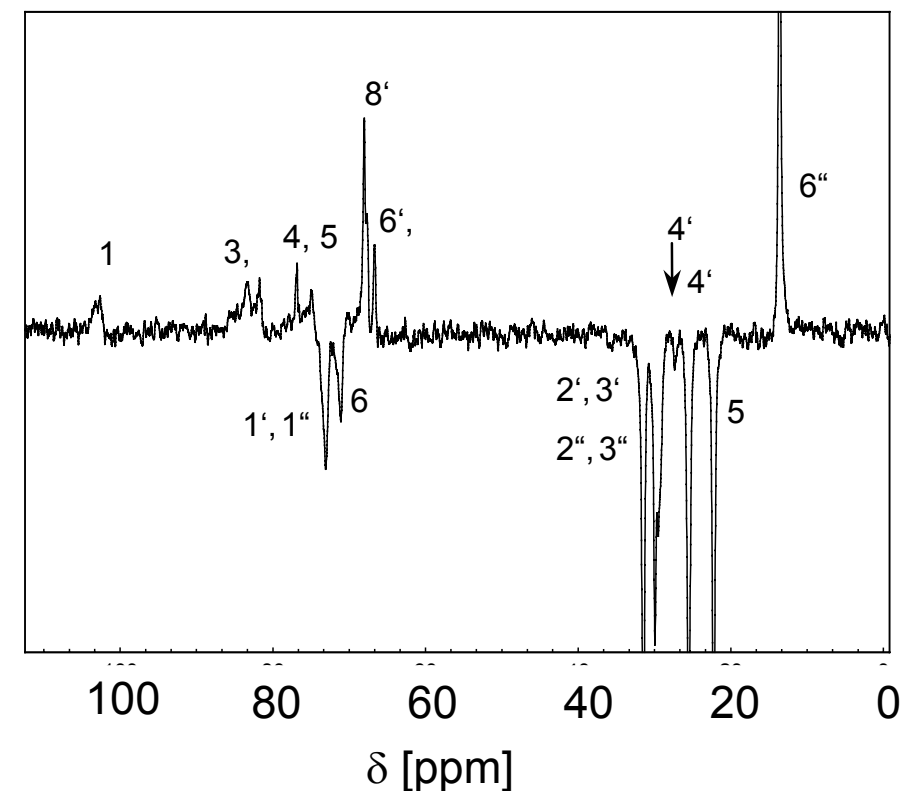

Fig. 6. DEPT spectrum of cellulose derivative $\mathbf{7 b}(6)$, recorded in $\mathrm{CDCl}_{3}$

Characteristic of all ${ }^{13} \mathrm{C}$ NMR spectra of the ferrocene-containing cellulose derivatives $\mathbf{7 b}$ is a shift of the carbon resonances towards higher fields in comparison to the corresponding absorptions of the pure $\mathrm{n}$-hexyl cellulose reference compounds $7 \mathrm{a}$ by approx. $\Delta \delta \approx 2.4-3.6 \mathrm{ppm}$ (relative to TMS as the internal standard). This shift is nearly independent of the ferrocene content in the respective cellulose derivative. This might be interpreted as the result of a quite homogeneous distribution of the ferrocene moieties along the cellulose chains: close neighbourhood of all constitutional units of the chains to ferrocenyl units could cause this shielding of the external magnetic field. 
In the IR spectra as well, there were observable all characteristic absorptions of the cellulose backbone and of the ether substituents of polymers $\mathbf{7 b}$. Moreover, absorptions around $3400 \mathrm{~cm}^{-1}$ were found to be nearly absent after careful drying in all cases where the $\mathrm{DS}_{\mathrm{fc}}$ was lower than approx. 1.5 (Fig. 7). This is a strong evidence for an almost complete conversion of the cellulose hydroxyl groups and thus a $\mathrm{DS}_{0}$ of approx. 3 in these materials. On the other hand, there was detectable a weak absorption in this region when the $\mathrm{DS}_{\mathrm{fc}}$ was greater than 1.5. This fact points towards some remaining free hydroxyl groups in these latter samples, potentially caused by steric conflicts in the cellulose repeating units which bear more than one ferrocenyl moiety. Hence, substitution of the third hydroxyl group might be retarded.

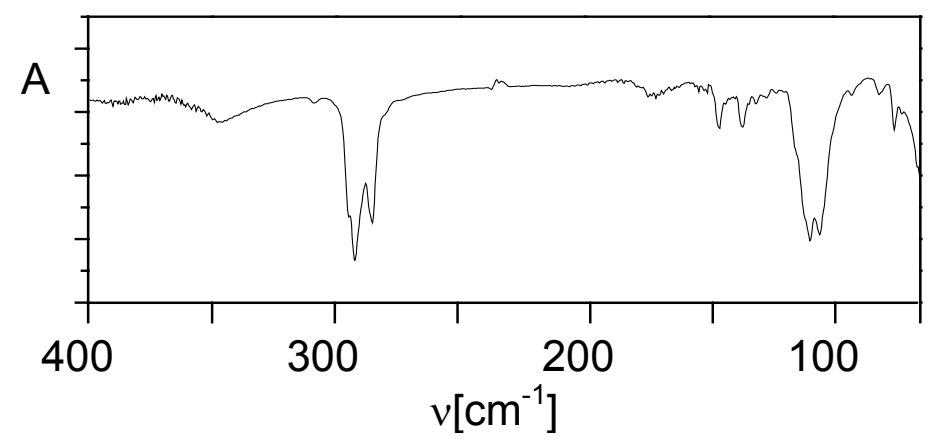

Fig. 7. IR spectrum of cellulose derivative $7 b(4)$

\section{Viscometry}

Viscosity investigations using the soluble fractions of the ferrocene-containing cellulose derivatives $7 \mathrm{~b}$ were carried out in chloroform solution at $30^{\circ} \mathrm{C}$. Fig. 8 shows the Huggins plot of a cellulose derivative having a $\mathrm{DS}_{\mathrm{fc}} \approx 0.2$ together with two plots of $n$-hexyl cellulose samples $7 a$. Comparison makes clear that the intrinsic viscosity of the ferrocene-containing cellulose derivative is clearly lower, by a factor of approx. 1.3 , as compared to the $n$-hexyl substituted derivative $7 \mathbf{a}\left(\mathrm{DS}_{\mathrm{fc}}=0\right)$.

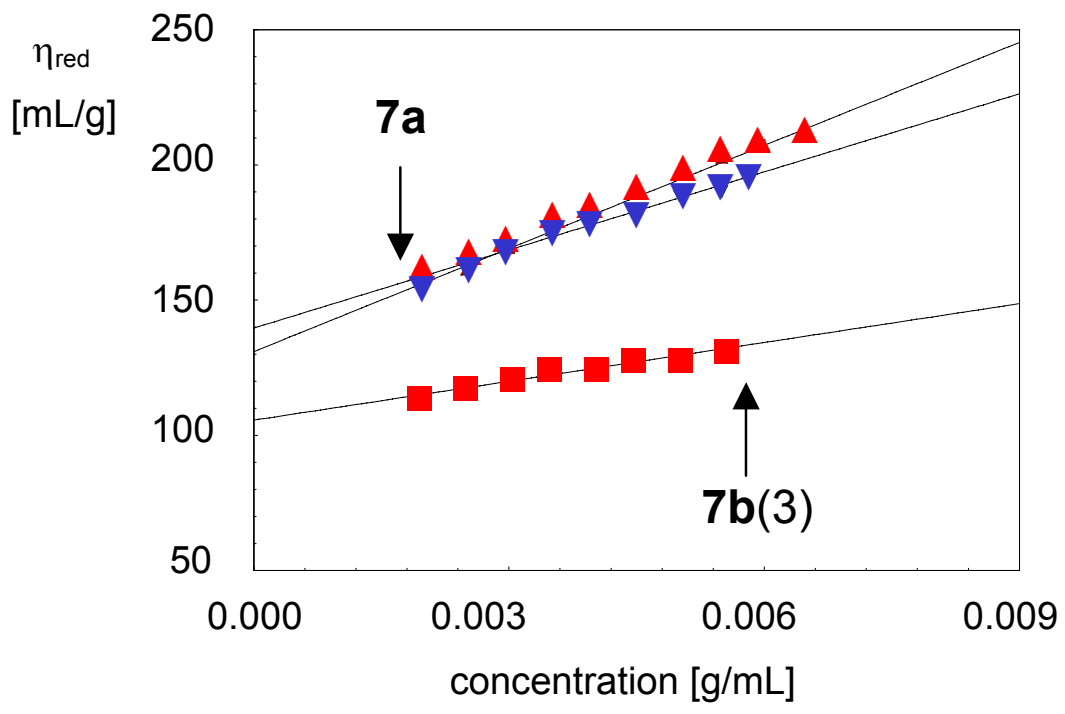

Fig. 8. Huggins plots of two n-hexyl cellulose derivatives 7 a and cellulose derivative $7 \mathbf{b}(3)$. All samples were produced under identical conditions; measurements were performed in $\mathrm{CHCl}_{3}$ at $30^{\circ} \mathrm{C}$ 
Altogether, it was a standard observation that, the higher the ferrocene content was in the cellulose derivative the lower was the intrinsic viscosity, even if samples were prepared under exactly identical conditions. This result might be explained by either more extensive chain degradation in the presence of the organometallic substituent, or by an intrinsically lower hydrodynamic volume of the latter derivative. Lowered hydrodynamic volumina caused by lateral substituents might be due to either some microphase separation - leading to an intramolecular clustering of the metallocene subunits and thus coil contraction - or due to remaining free hydroxyl groups - which undergo intramolecular hydrogen bonds and thus, again, coil contraction. The latter explanation seemed to be more probable because some hydroxyl groups were found by IR spectroscopy. Moreover, a further hint towards remaining hydroxyl groups also in the soluble material was that - while the viscometry measurements gave reliable results for all samples with $\mathrm{DS}_{\mathrm{fc}} \leq 1$ - the data points scattered in the case of elution time values when $D S_{f c}$ was greater than 1 - even if the solutions were optically clear. Maybe the low amount of hydroxyl groups in these samples does not prevent dissolution and filtration but is able to form microgels.

\section{Coupled size exclusion chromatography and light scattering investigations}

In order to analyze the above results in more detail, SEC experiments were carried out using an online light-scattering detector (SEC-MALLS). Such a combination of analytical methods gives the absolute molar mass $M$ for each fraction - and thus the true molar mass distribution curves. Simultaneously it is possible to determine the exponent $v$ of the scaling relation between the root of the mean square radius of gyration $\left\langle s^{2}\right\rangle^{1 / 2}$ and the molar mass:

$<s^{2}>^{1 / 2} \propto M^{v}$

Valuable information about the shape and the dimensions of the dissolved macromolecules can be obtained. For a coil dissolved under $\theta$ conditions, $v$ assumes $a$ value of 0.5 . For good solvents, $v$ takes a value between 0.5 and 0.6 while for rigid rods $v=1$. Very compact structures like (hyper)branched polymers, on the other hand, have $v<0.5$, approaching the hard sphere limit with $v=0.33$.

In order to determine $M_{\mathrm{w}}$ by light scattering, the refractive index increments $\mathrm{d} n / \mathrm{d} c$ were required for the soluble fractions of selected samples of 7 (Tab. 1). In Fig. 9 the obtained data are plotted vs. the experimentally determined ferrocene content of the respective sample 7 . From this plot it is evident that the values of $d n / d c$ increase linearly with increasing ferrocene content. This is an additional proof of the correctness of the $D S_{\mathrm{fc}}$ values determined by ${ }^{1} \mathrm{H}$ NMR.

Using SEC-MALLS coupling, the soluble fractions were chromatographically separated and simultaneously, the molar masses $M$ were determined for each chromatographic fraction. For the determination of $M$ and $\left\langle s^{2}\right\rangle^{1 / 2}$, the method of Debye was applied. Here, the values of $\mathrm{R}(\theta) / \mathrm{K} c$ are plotted vs. $\sin ^{2}(\theta / 2)$ for every fraction [32]. In Tab. 1 , the average values of $M_{\mathrm{w}}$ of some representative samples 7 are collected. From this table it is evident that the average molar masses of the samples do not show any systematic dependence on the $D S_{f c}$. Instead, the values scatter within a factor of 2. This reflects the difficulty to ensure constant chain degradation during the macromolecular substitution reaction. Also, the fractionation during the work-up procedure might lead to some differences, despite of much effort in optimization. 


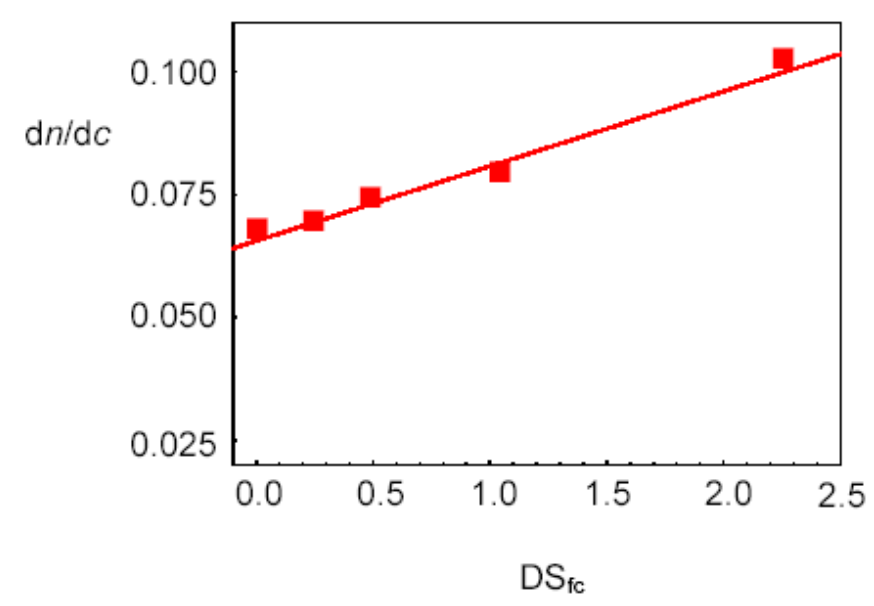

Fig. 9. Refractive index increments, $d n / d c$, of n-hexyl cellulose and a series of cellulose derivatives $7 \mathbf{b}$ in THF, plotted $v s$. DS $\mathrm{fc}_{\mathrm{fc}}(\mathrm{NMR})$

In Fig. 10, the $90^{\circ}$-light scattering traces (dotted lines) and the calibration curves obtained by SEC-MALLS (individual signs) are plotted for four selected ferrocenecontaining cellulose derivatives as well as for the n-hexyl cellulose reference. It is obvious that the values of $M$ of polymers 7 depend nearly linearly on the elution volume until a $\mathrm{DS}_{\mathrm{fc}}$ of approx. 1.5 is reached. If the ferrocene content increases further, this linearity is lost, and the plots show some upward curvature. For a pure SEC elution the slope of the calibration curve is related to $1 /(1+a)$ with a being the Mark-Houwink exponent. Thus, the increase in slope can be interpreted as a first hint towards a change to a more compact structure. In order to extract information about the shape of the cellulose derivatives in solution from these data, $\log \left(\left\langle s^{2}\right\rangle^{1 / 2}\right)$ was plotted vs. $\log M$ (Fig. 11).

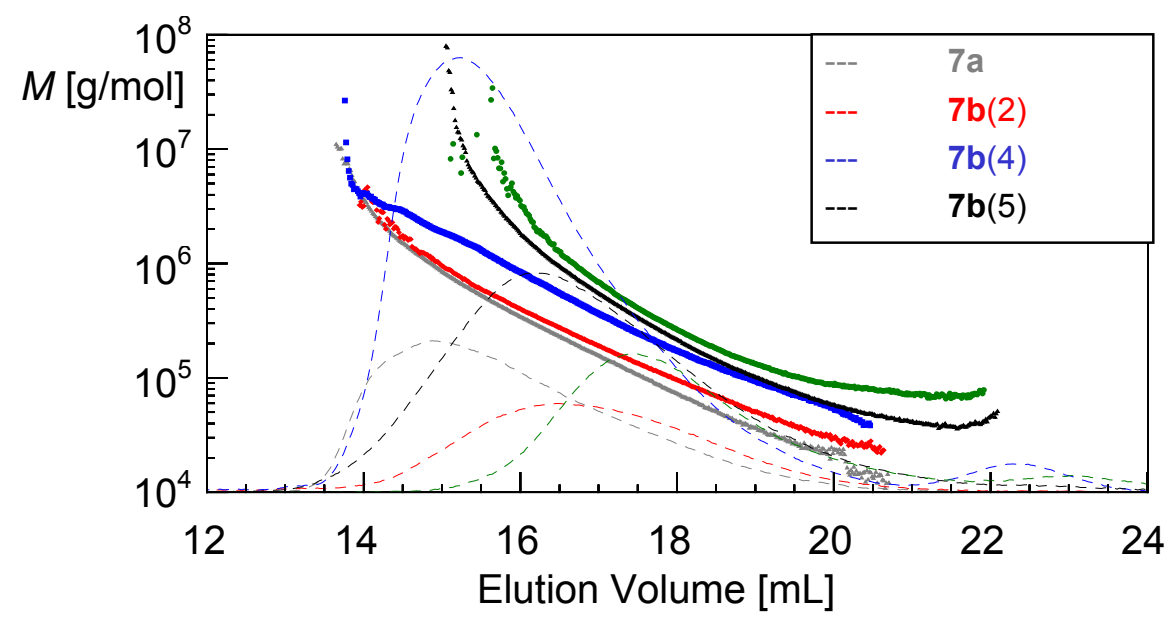

Fig. 10. Calibration curves and RI trace (dotted lines) for 5 cellulose ethers $\mathbf{7 a}$ and $\mathbf{7 b}$

At the high molar mass end of the chromatogram the refractive index (RI) detector losses intensity much faster than the light scattering signal due to its different molar mass dependence. The opposite is the case at the low molar mass region. Therefore the errors at the tails of the chromatograms are larger than in the central part. Furthermore, reliable determination of the mean squared radius of gyration is possible only for sufficiently large molecules $(>20 \mathrm{~nm})$. Otherwise the angular 
variation of the scattered light is to weak to be detectable. In the confidential measuring range $\left(2 \times 10^{5}=M_{\mathrm{w}}=2 \times 10^{6}\right)$, an almost linear dependence of $\left\langle s^{2}\right\rangle^{1 / 2}$ on $M$ is found for all samples.

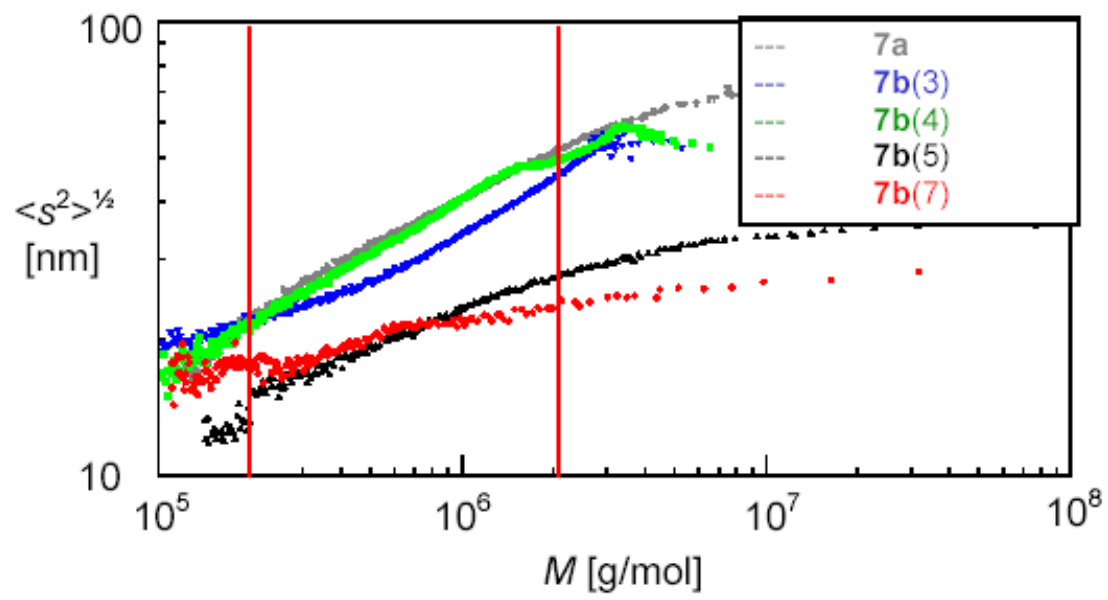

Fig. 11. Plot of $\left\langle s^{2}\right\rangle^{1 / 2}$ vs. the molar mass for five cellulose derivatives 7

In the plots shown in Fig. 11, no significant differences can be observed between the curves obtained from $n$-hexyl cellulose $7 a$ and ferrocene-containing cellulose derivatives $\mathbf{7 b}$ until $a \mathrm{DS}_{\mathrm{fc}}$ of approx. 1.0. For higher values of $D S_{\mathrm{fc}}$, the value of $\left\langle s^{2}\right\rangle^{1 / 2}$ for a certain molecular weight decreases clearly with increasing $D S_{\mathrm{fc}}$. This effect might be due to a change in the molecule's structure. This conclusion is further supported by the low value of the exponent $v$ of the $\left\langle s^{2}\right\rangle^{1 / 2}-M$ relation. Tab. 2 shows - in addition to the average radii of gyration $\left\langle s^{2}\right\rangle^{1 / 2}-$ also the values of $v$ determined from the slopes of the curves shown in Fig. 11.

Tab. 2. Average radii of gyration, $\left\langle s^{2}>^{1 / 2}\right.$, and slopes $v$ for cellulose derivatives

\begin{tabular}{l|llllll} 
& $\mathbf{7 a}$ & $\mathbf{7 b}(2)$ & $\mathbf{7 b}(3)$ & $\mathbf{7 b}(4)$ & $\mathbf{7 b}(5)$ & $\mathbf{7 b}(7)$ \\
\hline $\mathrm{DS}_{\mathrm{fc}}(\mathrm{NMR})$ & 0 & 0.2 & 0.5 & 1.0 & 1.4 & 2.3 \\
$M_{\mathrm{w}}$ in $\mathrm{g} / \mathrm{mol}$ & 200000 & 127000 & 354000 & 481000 & 253000 & 226000 \\
$\left\langle s^{2}>^{1 / 2} / \mathrm{nm}\right.$ & 41 & 23 & 37 & 42 & 22 & 20 \\
$v$ & 0.43 & 0.51 & 0.41 & 0.39 & 0.30 & 0.27
\end{tabular}

The slopes $v$ have values of 0.30 und 0.27 in the case of samples $7 \mathbf{b}(5)$ and $7 \mathbf{b}(7)$, respectively. These values of $v$ are clearly lower than those of the other compounds 7 : here, values of $v \approx 0.4-0.5$ were found which are similar to those of $n$-hexyl cellulose $7 \mathbf{a}$. These latter values correspond to readily dissolved cellulose derivatives [33-35]. This allows the conclusion that all the cellulose ethers having ferrocene contents of up to $\mathrm{DS}_{\mathrm{fc}}=1.0$ readily dissolve in THF solution. When the ferrocene contents are higher, the coils become more and more compact - in clear contradiction of the intuitive expectation where one would assume that the coils expand with increasing ferrocene content due to their high steric demands. On the contrary, however, the coils seem to approach compact spheres with increasing ferrocene content. 
In order to interpret the experimental results, we should recall that (i) for all cellulose derivatives $\mathbf{7 b}$ having values $\mathrm{DS}_{\mathrm{fc}}>1$ lower ferrocene contents were found in the polymers than that provided in the reagent mixture. Moreover, (ii) IR spectroscopy shows a low but - in most cases readily detectable - amount of hydroxyl groups in the ferrocene-containing polymers $\mathbf{7 b}$ - in contrast to the $n$-hexyl cellulose derivatives that were practically per-substituted. Also, (iii) this amount of free hydroxyl groups increases with increasing amount of ferrocene groups, i.e., $\mathrm{DS}_{\mathrm{fc}}$, in the polymer. Simultaneously, (iv) the amount of polymer found to be insoluble after work-up increases with increasing $D S_{f c}$ as well, starting from nearly $0 \%$ for $D S_{f c}=0$ up to $100 \%$ for $\mathrm{DS}_{\mathrm{fc}}=3$. These facts allow to conclude that the metallocene moieties attached to a cellulose backbone develop a quite large steric demand there affecting any further substitution of the remaining free hydroxyl groups. These hydroxyl groups form mainly intramolecular hydrogen bonds in the soluble product fractions, and the probability of free hydroxyl groups left in the final product increases with increasing ferrocene content. Therefore, an increasing $\mathrm{DS}_{\mathrm{fc}}$ does not lead to coil expansion but - due to the competitive effect of intramolecular hydrogen bonds that (over)compensate the steric demand of the substituents - to the observed coil collapse, and the hydrodynamic volume decreases with increasing ferrocene content at a constant chain length. This is in full agreement with the results of the viscosity experiments. Similar effects are known for partially substituted cellulose derivatives $[1,2,34]$. Here, $v$ can decrease down to 0.2 . Hence, values of $v$ smaller than 0.33 are understood as a consequence of hydrogen bond mediated aggregation.

Our explanation for free hydroxyl groups is the steric conflict caused by the bulky ferrocene moieties - for n-hexyl cellulose, no free hydroxyl groups were observed. Therefore, we tried to lower the steric demand of the ferrocene substituents by increasing the spacer length: n-hexyl spacers were introduced instead of the n-butyl spacers under discussion so far. However, the behaviour of the resulting polymers was similar to that of polymers $\mathbf{7 b}$ : there as well it was impossible to obtain cellulose derivatives having ferrocene-containing side chains attached to all hydroxyl groups. Again, these materials became greenish-black and insoluble after work-up. Thus, even longer spacers will be required for achieving the goal of a cellulose derivative having a $\mathrm{DS}_{\mathrm{fc}} \approx 3$, provided the explanations given above are valid. However, this is very probable while other explanations for the shrinking of the coils with increasing $\mathrm{DS}_{\mathrm{fc}}$ are rather improbable: one principal possibility might be the formation of helical structures. However, this can be excluded due to the irregularity of the substitution pattern generated here. Also, the formation of branches can be excluded. A further reason might be that, with increasing content of ferrocene in the cellulose derivative, there is a change of the aspect ratio (chain length vs. chain diameter). This effect, however, is insufficient to explain the results. Last but not least, it might be possible that a small amount of the ferrocene moieties is oxidized in every polymer obtained here. These oxidized substituents represent electrolyte functionalities, which can undergo ionic interactions and cause - in the organic solvent - coil contraction as well. However, no evidence was found for such partially oxidized sub-structures using NMR and UV-vis spectroscopy: only in the specific case where the ferrocene content should be $\mathrm{DS}_{\mathrm{fc}}=3$, discolouring was observed during work-up - in connection with the change into a completely insoluble material. This might point towards stabilization of mixed-valent states when ferrocene moieties are attached to cellulose in a very close neighbourhood, i.e., having three ferrocene substituents at every cellulose repeating unit. This close packing of redox-active metallocenes might force partial oxidation and formation of mixed-valent states but it seems not to be the explanation for the solution properties observed here for the other samples $\mathbf{7 b}$. 


\section{Conclusions and outlook}

In conclusion, introduction of 4-ferrocenylbutyl and n-hexyl side groups into cellulose backbones is possible by treating cellulose triacetate in $\mathrm{DMSO} / \mathrm{NaOH}$ solution with mixtures of 4-bromobutylferrocene and n-hexyl bromide. An almost quantitative conversion of the cellulose hydroxyl groups was reached when only one ferrocene substituent is attached to a cellulose repeating unit on average. At higher degrees of ferrocene substitution, some of the cellulose hydroxyl groups seem to remain unsubstituted. This conclusion has been drawn on the basis of SEC-MALLS experiments where coil contraction was found with increasing ferrocene content. It was further supported by IR spectroscopy, viscometry, and conventional SEC investigations. We assume steric hindrance of the bulky side groups and - in the case of very high degrees of ferrocene substitution - partial oxidation of the metallocenes to be responsible for incomplete conversion, preventing the formation of highly ferrocenesubstituted and nevertheless soluble products. Introduction of clearly longer spacer groups between the cellulose backbone and the lateral metallocene moieties could be an appropriate measure for obtaining cellulose derivatives having very high ferrocene content.

\section{Experimental part}

\section{Materials}

All chemicals and solvents were purchased from Acros, Aldrich, Fluka and Lancaster Chemical Co. and used without further purification unless otherwise stated. In order to remove peroxide impurities and oxygen, THF was refluxed with sodium or calcium hydride, and distilled under nitrogen.

\section{Methods}

NMR spectra were recorded at room temperature using a Bruker AM 400 NMR spectrometer working at $400 \mathrm{MHz}\left({ }^{1} \mathrm{H}\right)$ and $100 \mathrm{MHz}\left({ }^{13} \mathrm{C}\right)$, and a Bruker AM 500 NMR spectrometer working at $500 \mathrm{MHz}\left({ }^{1} \mathrm{H}\right)$ and $125 \mathrm{MHz}\left({ }^{13} \mathrm{C}\right)$. The signal assignment is done according to the numbering given in Scheme 3. Multiplicities are given as ' $s$ ' for singlet, ' $d$ ' for doublet, 't' for triplet, ' $q$ ' for quartet, and ' $m$ ' for multiplet. TMS was the internal standard. IR spectra were recorded on a Shimadzu FTIR-810M as films, prepared from chloroform solution on $\mathrm{KBr}$ plates under nitrogen.

SEC chromatograms were recorded in THF (100 ppm toluene as internal flow marker) on a system consisting of a Waters 510 pump, a TSP AS 100 injector and PL Mixed bed columns (PL Gels B, C and D, $30 \times 0.78 \mathrm{~cm}$ each) (Polymer Laboratories, Shropshire, UK). Detection was achieved using a Waters RI 410 differential refractometer, a Waters UV-vis 486 detector and a Wyatt Technology Dawn EOS MALLS detector $(\lambda=690 \mathrm{~nm})$. The solutions were filtered through PTFE filters (Acrodish GF, $0.45 \AA$ ). . Conventional SEC data were collected using the WINGPC V. 6.01 software. Polystyrene is used for calibration. SEC-MALLS data were acquired using Winastra version 4.7.3 (Wyatt Technology, Santa Barbara, USA). The refractive index increments were determined at $\lambda=633 \mathrm{~nm}$ using a ScanRef instrument of the PSS company, Mainz, Germany.

Elemental analyses were carried out using a Perkin Elmer 240B instrument and a $\mathrm{CHN}$-Rapid instrument of Elementar Analysensysteme. Viscosity investigations were 
carried out on a PVS 2.03 of the Lauda company, using an Ubbelohde capillary viscometer (type Oc). Chloroform was the solvent; the temperature was $30^{\circ} \mathrm{C}$.

\section{Ferrocenyl-3-bromopropyl ketone [31]}

In a $50 \mathrm{~mL}$ reaction flask, 4-bromobutyric chloride $(2 \mathrm{~mL}, 0.017 \mathrm{~mol}), \mathrm{AlCl}_{3}(2.3 \mathrm{~g}$, $0.017 \mathrm{~mol})$ and $\mathrm{CH}_{2} \mathrm{Cl}_{2}(20 \mathrm{~mL})$ were stirred under an atmosphere of nitrogen at $0^{\circ} \mathrm{C}$ for $20 \mathrm{~min}$. The slightly yellowish solution was transferred into a dropping funnel and slowly added to a cooled $\left(0^{\circ} \mathrm{C}\right)$ solution of ferrocene $(3.2 \mathrm{~g}, 0.017 \mathrm{~mol})$ in $\mathrm{CH}_{2} \mathrm{Cl}_{2}(30$ $\mathrm{mL}$ ). During that, the colour changed from orange to reddish brown. After stirring the reaction mixture for $3 \mathrm{~h}$ at room temperature, water $(50 \mathrm{~mL})$ was carefully added. The phases were separated, and the organic layer was washed with water until the aqueous layer remained neutral. The organic layer was dried (MgSO4), filtered, and the solvent was removed in vacuum. The residue was dried in vacuum and then redissolved in chloroform and filtered over a column of silica gel. Dark red oil was obtained (3.57 g, $0.0107 \mathrm{~mol}, 63 \%$ (lit.: 85\%)).

${ }^{1} \mathrm{H} \mathrm{NMR}\left(\mathrm{CDCl}_{3}\right): \delta=4.83(\mathrm{~s}, 2 \mathrm{H}, \mathrm{H} 2), 4.53(\mathrm{~s}, 2 \mathrm{H}, \mathrm{H} 3), 4.24(\mathrm{~s}, 5 \mathrm{H}, \mathrm{H} 4), 3.59(\mathrm{t}, 2 \mathrm{H}$, $\mathrm{H} 8), 2.96$ (t, 2H, H6), $2.28(\mathrm{t}, 2 \mathrm{H}, \mathrm{H} 7)$.

${ }^{13} \mathrm{C} \mathrm{NMR}\left(\mathrm{CDCl}_{3}\right): \delta=202.96$ (C5), 79.21 (C1), 72.78 (2), 70.32 (C4), 69.71 (C3), 37.61 (C6), 34.51 (C8), 27.32 (C7).

HRMS ( $\mathrm{C}_{14} \mathrm{H}_{15} \mathrm{FeOBr}$ : 335.0229): 333.9667; MS (70 eV, El, 60 $\left.{ }^{\circ}\right)$ : 334,0 (100 [M]+).

\section{4-Ferrocenyl-n-butyl bromide [31]}

In a $250 \mathrm{~mL}$ reaction flask, $\mathrm{HgCl}_{2}(0.6 \mathrm{~g})$, zinc powder $(7 \mathrm{~g})$, ferrocenyl 3-bromopropyl ketone $(3.57 \mathrm{~g}, 0.0107 \mathrm{~mol})$, toluene $(30 \mathrm{~mL})$ and water $(25 \mathrm{~mL})$ were added. Subsequently, after some stirring, concentrated hydrochloric acid $(20 \mathrm{~mL})$ was added. The reaction mixture was stirred under reflux for $4 \mathrm{~h}$. After cooling down to room temperature, the layers were separated off, and the aqueous layer was washed with some toluene. The combined organic layers were dried $\left(\mathrm{MgSO}_{4}\right)$. The solid was filtered off, the $\mathrm{MgSO}_{4}$ residue was washed with some toluene, and the organic layer was concentrated down in vacuum. The residue was dried in vacuum. Yield: $2.15 \mathrm{~g}$ $(0,0067 \mathrm{~mol}, 80 \%$ (lit.: $90 \%))$ of an intensely reddish-orange oil.

${ }^{1} \mathrm{H}$ NMR $\left(\mathrm{CDCl}_{3}\right): \delta=1.66(\mathrm{~m}, 2 \mathrm{H}, \mathrm{H} 6) ; 2.05$ (q, 2H, H7); $2.36(\mathrm{t}, 2 \mathrm{H}, \mathrm{H} 5) ; 3.43(\mathrm{t}, 2 \mathrm{H}$, $\mathrm{H} 8) ; 4.06$ (s, 4H, H2/3); 4.11 (s, 5H, H4).

${ }^{13} \mathrm{C} \mathrm{NMR}\left(\mathrm{CDCl}_{3}\right): \delta=28.68$ (s, C5); 29.48 (s, C6); 32.50 (s, C7); 33.76 (s, C8); 67.29 (C3); 68.16 (C2); 68.65 (s, C4); 88.73 (s, C1).

HRMS $\left(\mathrm{C}_{16} \mathrm{H}_{17} \mathrm{FeBr}\right)$ : calc.: 321.0393, found: 319.9854; MS (70 eV, El, $\left.20^{\circ} \mathrm{C}\right): 320.1$ $(100[\mathrm{M}]+)$.

\section{Cellulose ethers 7}

Under an atmosphere of nitrogen, cellulose triacetate $(1 \mathrm{~g}, 3.47 \mathrm{mmol})$ was stirred for $1 \mathrm{~h}$ at $70^{\circ} \mathrm{C}$ in dry DMSO $(100 \mathrm{~mL})$. After cooling down to room temperature, powdered $\mathrm{NaOH}$ (5 g, 0.125 mol, 12 eqiv. pro $\mathrm{OH}$ function) was added. After $1 \mathrm{~h}$ stirring at room temperature, the alkyl halogenides (12 eqiv.) were added slowly. The mixture was stirred 8 days at room temperature. Water $(400 \mathrm{~mL})$ was added, and the 
resulting mixture was extracted using $\mathrm{CH}_{2} \mathrm{Cl}_{2}(5 \times 200 \mathrm{~mL})$. The combined organic layers were dried and concentrated down to a few milliliters. Methanol was added slowly until the polymer precipitated. The solid was isolated, dried, and redissolved in methylene chloride $(50 \mathrm{~mL})$. Again, methanol was added to the resulting solution to precipitate the polymer, which was finally dried in vacuum $\left(50^{\circ} \mathrm{C}, \mathrm{P}_{4} \mathrm{O}_{10}\right)$. Yield: approx. $75 \%$.

\section{n-Hexyl model celluloses 7a}

${ }^{1} \mathrm{H} \mathrm{NMR}\left(\mathrm{CDCl}_{3}\right): \delta=0.74\left(\mathrm{~s}, 3 \mathrm{H}, \mathrm{H6} 6^{\prime}\right) ; 1.13\left(\mathrm{H}^{\prime}-\mathrm{H}^{\prime}\right) ; 1.38$ (2'); 1.91 (s, H2'); 3.8 2.7 (br, 5H, H1-H5); 4.18 (s, 2H, H6).

${ }^{13} \mathrm{C} \mathrm{NMR}\left(\mathrm{CDCl}_{3}\right): \delta=13.33$ (s, C6'); 21.91 (s, C5'); 25.17 (s, C4'); 29.65 (s, C3'); 31.15 (s, C2'); 68.6 (br, C6); 71.43 (s, C1'); 73.48 (s, C1'); 75.33 (s, C5); 77.23 (s, C4); 82.20 (s, C2); 83.72 (s, C3); 103.03 (s, C1).

\section{Ferrocene-containing cellulose derivatives $\mathbf{7 b}$}

${ }^{1} \mathrm{H}$ NMR $\left(\mathrm{CDCl}_{3}\right): \delta=0.89$ (s, H6“); 2.6 - 1.1 (br, H2'-H4', H2“-5“); 4.6 - 2.8 (br, H1', H1“, H6'-8', H1-6).

${ }^{13} \mathrm{C}$ NMR $\left(\mathrm{CDCl}_{3}\right): \delta=13.79$ (C6"); 22.30 (C5"); 26.65 (C4"); 27.52 (C4'); 30.08, 29.94 (C3', C3"); 31.62 (C2', C2"); 67.84, 66.86 (C6', C7'); 68.18 (C8'); 73.23, 71.25 (C6, C1', C1"); 76.98, 75.14 (C4, C5); 81.82 (C2); 83.43 (C3); 89.08 (C5'); 103.13 (C1).

Acknowledgement: The authors wish to thank Deutsche Forschungsgemeinschaft (DFG) and Fonds der Chemischen Industrie (FCl) for financial support.

[1] Klemm, D.; Philipp, B.; Heinze, T.; Heinze, U.; Wagenknecht, W.; "Comprehensive Cellulose Chemistry", vol. 1, 'Fundamentals and Analytical Methods', Wiley-VCH, Weinheim 1998.

[2] Klemm, D.; Philipp, B.; Heinze, T.; Heinze, U.; Wagenknecht, W.; "Comprehensive Cellulose Chemistry”, vol. 2, 'Functionalization of Cellulose', Wiley-VCH, Weinheim 1998.

[3] "Cellulose Derivatives - Modification, Characterization, and Nanostructures", Heinze, T.; Glaser, W.; editors; ACS Symp. Ser. 688, 1998.

[4] Schmidt, B.; Bioforum 2000, 1, 19.

[5] Ruck, H.; Das Papier 1986, 40, 93, 153.

[6] Desai, N. B.; Wisotzki, K.; Seifen-Öle-Fette-Wachse 1991, 117, 124.

[7] Burchard, W., editor; "Polysaccharide", Springer, Berlin 1985.

[8] Kondo, T.; J. Polym. Sci. 1994, B32, 1229.

[9] Brandt, L.; in "Ullmann's Encyclopedia of Industrial Chemistry", Gerhartz, W., editor; $5^{\text {th }}$ edition, vol. $A 5, \mathrm{VCH}$, Weinheim 1986.

[10] Dönges, R.; Das Papier 1997, 51, 653. 
[11] Löscher, F.; Ruckstuhl, T.; Jaworek, T.; Wegner, G.; Seeger, S.; Langmuir 1998, 14, 2786.

[12] Basque, P.; de Gunzbourg, A.; Rondeau, P.; Ritcey, A. M.; Langmuir 1996, 12, 5614.

[13] Fakirov, C.; Lieser, G.; Wegner, G.; Macromol. Chem. Phys. 1997, 198, 3407.

[14] Li, X. G.; Huang, M. R.; Hu, L.; Lin, G.; Yang, P. C.; Eur. Polym. J. 1999, 35, 157.

[15] Hudson, S. H.; Cuculo, J. A.; J. Macromol. Sci. - Rev. Macromol. Chem. 1980, C18, 1.

[16] Felcht, U. H.; in "Cellulose and its Derivatives - Chemistry, Biochemistry and its Applications", Kennedy, J. F.; Phillips, G. O.; Wedlock, D. J.; Williams, P. A.; editors; Ellis Horwood Ltd, Chichester 1985.

[17] Heinze, T.; Erler, U.; Nehls, I.; Klemm, D.; Angew. Makromol. Chem. 1994, 215, 93.

[18] Turbak, A. F.; Hammer, R. B.; Davies, R. E.; Hergert, H. L.; Chemtech 1980, 51.

[19] Klüfers, P.; Burchard, W.; Habermann, N.; Seger, B.; Wilhelm, U.; Angew. Chem. 1994, 106, 936.

[20] Hanemann, O.; Dissertation, Karlsruhe University 1996.

[21] Saalwächter, K.; Burchard, W.; Klüfers, P.; Kettenbach, G.; Mayer, P.; Klemm, D.; Dugarmaa, S.; Macromolecules 2000, 33, 4094.

[22] McCormick, C. L.; Callais, P. A.; Hutchinson Jr., B. H.; Macromolecules 1985, 18, 2394.

[23] Schleicher, H.; Philipp, B.; Kabrelian, V.; Das Papier 1988, 42, 653.

[24] Dawsey, T. R.; McCormick, C. L.; J. Macromol. Sci. - Rev. Macromol. Chem. Phys. 1990, C30, 405.

[25] Kondo, T.; Gray, D. C.; J. Appl. Polym. Sci. 1992, 45, 417.

[26] Schuldt, U.; Philipp, B.; Klemm, D.; Stein, A.; Jancke, H.; Nehls, I.; Das Papier 1994, 48, 3.

[27] Kondo, T.; Carbohydr. Res. 1993, 238, 231.

[28] Kondo, T.; Gray, D. G.; Carbohydr. Res. 1991, 220,173.

[29] Klemm, D.; Schnabelrauch, M.; Stein, A.; Heinze, T.; Erler, U.; Vogt, S.; Das Papier 1991, 45, 773.

[30] Seufert, M.; Schaub, M.; Wenz, G.; Wegner, G.; Angew. Chem. 1995, 107, 363.

[31] Creager, S. E.; Rowe, G. K.; J. Electroanal. Chem. 1994, 370, 203.

[32] Mori, S.; Barth, H. G.; "Size Exclusion Chromatography”, Springer, Berlin 1999.

[33] Schulz, L.; Burchard, W.; Das Papier 1993, 47, 1.

[34] Kirste, R. G.; Wunderlich, W.; Zeitschr. Phys. Chem. 1968, 58, 133.

[35] Kasabo, F.; Kanematsu, T.; Nakagawa, T.; Sato, T.; Macromolecules 2000, 33, 2748. 